\title{
Leptin action in the forebrain regulates the hindbrain response to satiety signals
}

\author{
Gregory J. Morton, ${ }^{1}$ James E. Blevins, ${ }^{2}$ Diana L. Williams, ${ }^{1}$ Kevin D. Niswender, ${ }^{1}$ \\ Richard W. Gelling, ${ }^{1}$ Christopher J. Rhodes, ${ }^{3}$ Denis G. Baskin, ${ }^{2,4}$ and Michael W. Schwartz ${ }^{1}$

\begin{abstract}
1Department of Medicine, Harborview Medical Center and University of Washington, Seattle, Washington, USA. 2Veterans Affairs Puget Sound
\end{abstract} \\ Health Care System, Seattle, Washington, USA. ${ }^{3}$ Pacific Northwest Research Institute and Department of Pharmacology, University of Washington, \\ Seattle, Washington, USA. "Departments of Medicine and Biological Structure, University of Washington, Seattle, Washington, USA.
}

\begin{abstract}
The capacity to adjust energy intake in response to changing energy requirements is a defining feature of energy homeostasis. Despite the identification of leptin as a key mediator of this process, the mechanism whereby changes of body adiposity are coupled to adaptive, short-term adjustments of energy intake remains poorly understood. To investigate the physiological role of leptin in the control of meal size and the response to satiety signals, and to identify brain areas mediating this effect, we studied Koletsky $\left(f a^{k} / f a^{k}\right)$ rats, which develop severe obesity due to the genetic absence of leptin receptors. Our finding of markedly increased meal size and reduced satiety in response to the gut peptide cholecystokinin (CCK) in these leptin receptor-deficient animals suggests a critical role for leptin signaling in the response to endogenous signals that promote meal termination. To determine if the hypothalamic arcuate nucleus (ARC) (a key forebrain site of leptin action) mediates this leptin effect, we used adenoviral gene therapy to express either functional leptin receptors or a reporter gene in the area of the ARC of $f a^{k} / f a^{k}$ rats. Restoration of leptin signaling to this brain area normalized the effect of CCK on the activation of neurons in the nucleus of the solitary tract and area postrema, key hindbrain areas for processing satiety-related inputs. This intervention also reduced meal size and enhanced CCK-induced satiety in $f a^{k} / f a^{k}$ rats. These findings demonstrate that forebrain signaling by leptin, a long-term regulator of body adiposity, limits food intake on a meal-to-meal basis by regulating the hindbrain response to short-acting satiety signals.
\end{abstract}

\section{Introduction}

The discovery of leptin (1) and the hypothalamic neurons on which it acts (2-6) has begun to clarify how information regarding body energy stores is communicated to the brain and is subsequently "transduced" into behavioral and metabolic responses $(7,8)$. Much of this progress is due to the identification of specific neurons in the arcuate nucleus (ARC) of the hypothalamus that serve as sensors of whole-body energy status and initiate downstream responses designed to maintain fuel stores at a constant level $(7,8)$. Although many regions of the brain are involved in energy homeostasis, circuits that begin in the ARC are some of the best understood at the molecular level (9-11). Despite this progress, little is known about how the hypothalamic actions of leptin ultimately influence feeding behavior on a meal-to-meal basis.

The consumption of food during single meals is governed by mechanisms that act over short time intervals to control the initiation and termination of food ingestion. Whereas the timing of meal initiation is sensitive to a variety of both intrinsic and extrinsic factors (e.g., time of day, social and emotional factors, and food availability) and is consequently quite variable, meal termination is a more reproducible, biologically determined process (12). Central to the mechanism underlying meal termination is the perception

Nonstandard abbreviations used: AgRP, agouti-related peptide; AP, area postrema; ARC, arcuate nucleus; cFLI, c-Fos-like immunoreactivity; CCK, cholecystokinin; LHA, lateral hypothalamic area; NPY, neuropeptide Y; NTS, nucleus of the solitary tract; PFA, paraformaldehyde; POMC, pro-opiomelanocortin; PVN, paraventricular nucleus; VMN, ventromedial nucleus;

Conflict of interest: The authors have declared that no conflict of interest exists.

Citation for this article: J. Clin. Invest. 115:703-710 (2005).

doi:10.1172/JCI200522081. of satiety arising from neural and hormonal signals that are generated during food consumption, including gastric distension and secretion of the gut peptide cholecystokinin (CCK) (13). These and other stimuli activate vagal afferent fibers that terminate in the nucleus of the solitary tract (NTS), situated in the caudal hindbrain. Activation of neurons in the NTS and the adjacent area postrema (AP) by satiety signals, in turn, play an essential role in slowing gastric emptying and in promoting meal termination (14).

Several observations suggest that leptin reduces food intake by heightening the response to meal-related satiety signals. For example, pharmacological administration of leptin reduces meal size (15-18) and augments the response to CCK (19-21), whereas genetic or acquired reductions in leptin signaling reduce sensitivity to satiety cues (22-24). To explain how leptin action is coupled to the perception of satiety, we and others hypothesized that ARC neurons convey leptin-generated responses to the NTS via a descending projection from hypothalamus to hindbrain $(7,25-27)$. Because leptin receptors are also present in the hindbrain $(28,29)$, however, interactions between leptin and satiety signals such as CCK could potentially involve local actions that are independent of forebrain input.

The studies here were undertaken to clarify the physiological role played by leptin in the response to meal-related satiety signals and to identify brain areas that mediate this leptin effect. Specifically, we wished to test the hypothesis that leptin signaling in the forebrain (e.g., in the ARC) controls meal size by modulating the hindbrain response to satiety signals such as CCK. To accomplish this goal, we studied Koletsky $\left(f a^{k} / f a^{k}\right)$ rats, which develop hyperphagia and severe obesity due to the genetic absence of leptin receptors $(30,31)$. We hypothesized that if leptin signaling is an important determinant of the response to satiety signals, these animals should 

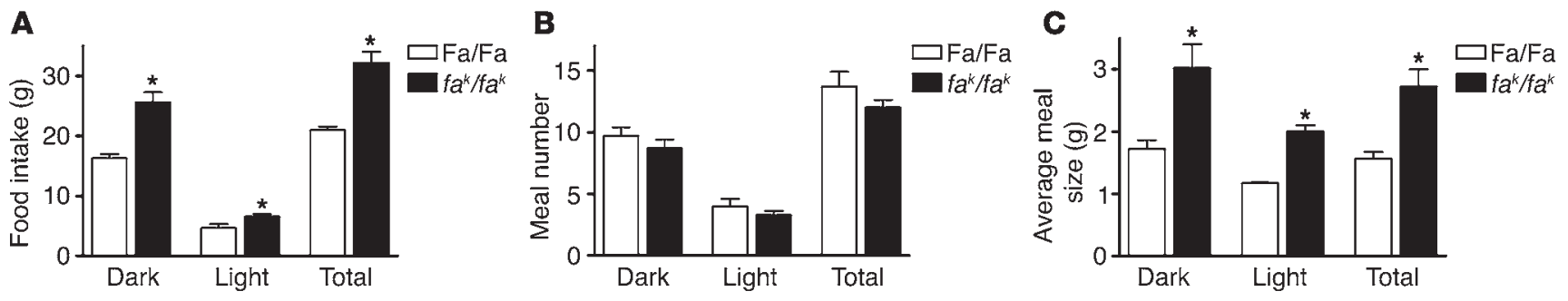

Figure 1

Effect of leptin receptor deficiency on meal patterns. Total food intake (A), meal number (B), and average meal size (C) of Fa/Fa (white bars) and $f a^{k} / f a^{k}$ (black bars) animals. Data are based on food intake measured continuously over a 24-hour period in rats housed individually in metabolic cages. ${ }^{*} P<0.05$.

both consume larger meals and display blunted hindbrain responses to CCK compared with those of their lean $(\mathrm{Fa} / \mathrm{Fa})$ littermates. Moreover, we reasoned that if hypothalamic neurons link afferent input from leptin to the control of these responses, restoration of leptin signaling to the hypothalamus of $f a^{k} / f a^{k}$ rats should both reduce meal size and enhance the response to satiety signals. Our findings support those predictions and identify the mediobasal hypothalamus as a key brain area that links leptin action to both the size of individual meals and the hindbrain response to CCK. Leptin-mediated communication between forebrain (ARC) and hindbrain (NTS) neurons provides a paradigm for understanding how changes of body fat mass elicit compensatory adjustments of food intake on a meal-to-meal basis.

\section{Results}

Experiment 1: effect of leptin receptor deficiency and ARC-directed leptin receptor gene therapy on meal patterns. To determine the effect of leptin receptor deficiency on meal patterning, we housed male $f a^{k} / f a^{k}$ rats and their lean $(\mathrm{Fa} / \mathrm{Fa})$ littermates (body weight, $494 \pm 6 \mathrm{~g}$ vs. $355 \pm 6 \mathrm{~g}$; $P<0.05)$ individually in metabolic cages equipped to continuously measure intake of standard chow. As expected, the $f a^{k} / f a^{k}$ animals exhibited a marked increase of food intake (by 53\%) over 24 hours relative to the $\mathrm{Fa} / \mathrm{Fa}$ controls $(32.3 \pm 1.9$ g vs. $21.1 \pm 0.6 \mathrm{~g} ; P<0.05)$ (Figure 1A). Food consumption by $f a^{k} / f a^{k}$ rats was increased relative to that of the $\mathrm{Fa} / \mathrm{Fa}$ controls during both the dark phase $(25.7 \pm 1.7 \mathrm{~g} v \mathrm{~s} .16 .4 \pm 0.7 \mathrm{~g} ; P<0.05)$ and the light phase $(6.60 \pm 0.38 \mathrm{~g}$ vs. $4.67 \pm 0.65 \mathrm{~g} ; P<0.05)$ of the night-day cycle. This effect was not attributable to an increase in meal number, as neither the total number of meals consumed (Figure 1B) nor the intermeal interval was significantly different between the two genotypes. In contrast, the average size of meals consumed by $f a^{k} / f a^{k}$ rats was $75 \%$ greater than that consumed by $\mathrm{Fa} / \mathrm{Fa}$ animals $(2.59 \pm 0.33 \mathrm{~g}$ vs. $1.48 \pm 0.16 \mathrm{~g}$; $P<0.05)$ (Figure 1C). Hyperphagic feeding in rats lacking leptin receptors is therefore characterized by the consumption of a normal number of excessively large meals.

To determine the effect of ARC-directed leptin receptor gene therapy on these parameters, we repeated the studies described above in a separate group of $f a^{k} / f a^{k}$ rats 6 days after microinjection of adenovirus expressing either the long form of the leptin receptor (Ad-LEPR-B) or a reporter gene (Ad-lacZ) into this brain area ( $n=5 /$ group). At this time point, there was no statistical difference in body weight $(541 \pm 11 \mathrm{~g}$ vs. $547 \pm 7 \mathrm{~g} ; P=\mathrm{NS})$ or weight loss $(-10.3 \pm 2.9$ g vs. $-1.4 \pm 4.4 \mathrm{~g} ; P=\mathrm{NS})$ between animals treated with Ad-LEPR-B and those treated with Ad-GFP. Consistent with our previous findings (32), 24-hour food intake was reduced (by $22 \%)$ in Ad-LEPR-B- compared with Ad-lacZ-treated animals $(20.4 \pm 0.9$ g vs. $26.1 \pm 0.9$ g; $P<0.05)$ (Figure 2A). As Ad-LEPR-Band Ad-lacZ-treated $f a^{k} / f a^{k}$ rats consumed comparable numbers of meals $(13.2 \pm 0.9 \mathrm{~g}$ vs. $11.4 \pm 0.9 \mathrm{~g} ; P=\mathrm{NS}$ ) (Figure $2 \mathrm{~B})$ that were separated by similar intermeal intervals (data not shown), the reduction in food intake resulting from leptin receptor gene therapy was attributable to a reduction in average meal size $(1.56 \pm 0.08$ g vs. $2.33 \pm 0.15 \mathrm{~g} ; P<0.05)$ (Figure 2C).

Experiment 2: Effect of leptin receptor deficiency and ARC-directed leptin receptor gene therapy on CCK-induced satiety. To identify an appropriate dose of CCK with which to determine the effect of leptin receptor deficiency and ARC-directed leptin receptor gene therapy on CCK-induced satiety, we first determined the dose-response curve. As expected, $f a^{k} / f a^{k}$ rats were hyperphagic relative to their $\mathrm{Fa} / \mathrm{Fa}$ littermates $(5.13 \pm 0.30 \mathrm{~g}$ vs. $3.50 \pm 0.23 \mathrm{~g} ; P<0.05)$ after i.p. vehicle injection. While CCK reduced food intake in rats of both genotypes, the magnitude of this anorexic effect was increased in $\mathrm{Fa} / \mathrm{Fa}$ animals,
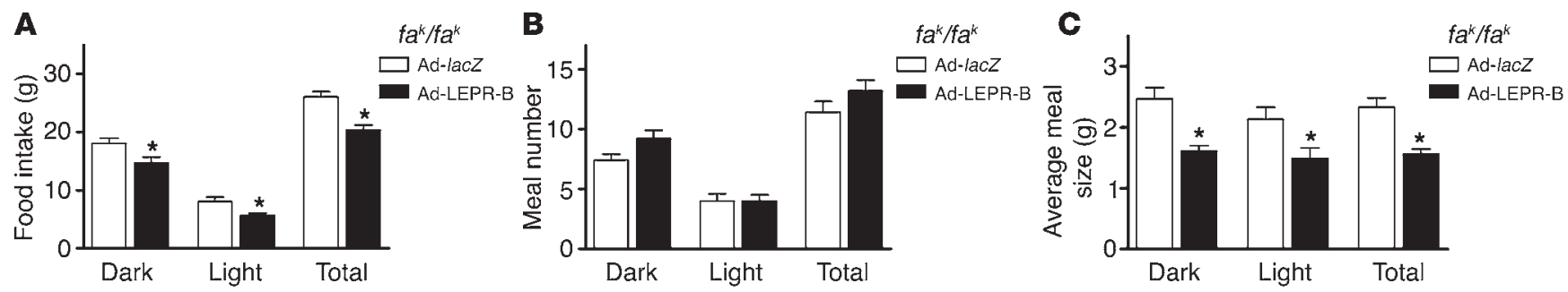

Figure 2

Effect of ARC-directed leptin receptor gene therapy on meal patterns. Total food intake (A), meal number (B), and average meal size (C) of $\mathrm{fa}^{k} / f \mathrm{f}^{k}$ rats, measured 6 days after ARC-directed microinjection of either Ad-lacZ (white bars) or Ad-LEPR-B (black bars). Data were collected continuously over a 24 -hour period. ${ }^{\star} P<0.05$. 


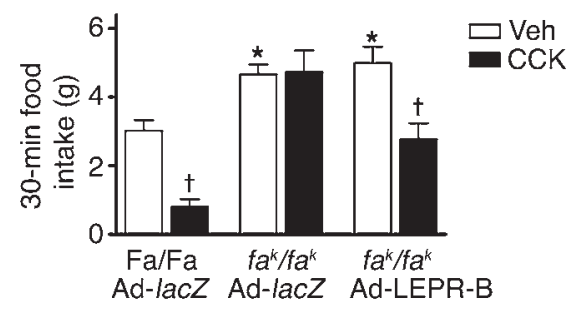

Figure 3

Effect of ARC-directed leptin receptor gene therapy on CCK-induced satiety. Thirty-minute food intake after i.p. injection of either saline vehicle (Veh; white bars) or a 1.0- $\mu$ g dose of CCK (black bars) in Fa/Fa or $\mathrm{fa}^{k} / \mathrm{fa} \mathrm{a}^{k}$ rats 6 days after microinjection of either Ad-lacZ or Ad-LEPR-B into the ARC. ${ }^{*} P<0.05$, vehicle injection in $\mathrm{Fa} / \mathrm{Fa}$ vs. $f a^{k} / f a^{k}$ rats; ${ }^{\dagger} P<0.05$, vehicle vs. CCK.

with doses of $0.75 \mu \mathrm{g}, 1.0 \mu \mathrm{g}$, and $1.5 \mu \mathrm{g}$ inhibiting 30 -minute feeding by $61 \%, 68 \%$, and $81 \%$, respectively, in $\mathrm{Fa} / \mathrm{Fa}$ animals compared with $36 \%, 44 \%$, and $59 \%\left(P<0.05\right.$ for each) in $f a^{k} / f a^{k}$ littermates. At higher doses of CCK $(5 \mu \mathrm{g})$, however, differences in the response were no longer significant, with CCK inhibiting 30-minute food intake by $89 \%$ and $90 \%$ in $\mathrm{Fa} / \mathrm{Fa}$ and $f a^{k} / f a^{k}$ animals, respectively.

In $\mathrm{Fa} / \mathrm{Fa}$ rats that received control adenovirus (Ad-lacZ), CCK injection $(1.0 \mu \mathrm{g}$ i.p.) robustly reduced 30 -minute food intake relative to saline injection $(0.80 \pm 0.22 \mathrm{~g}$ vs. $3.02 \pm 0.31 \mathrm{~g} ; P<0.05)$ (Figure 3). In contrast, the intake of $f a^{k} / f a^{k}$ rats treated similarly was not significantly affected by administration of this low dose of CCK. Among $f a^{k} / f a^{k}$ rats injected with saline i.p., 30-minute intake was not different between those treated with Ad-lac $Z$ versus those treated with Ad-LEPR-B ( $4.66 \pm 0.29$ g vs. $4.99 \pm 0.48 \mathrm{~g} ; P=\mathrm{NS})$. However, ARC-directed Ad-LEPR-B injection conferred upon $f a^{k} / f a^{k}$ rats the ability to respond to the satiety effect of this same dose of CCK. Thus, unlike Ad-lacZ-treated $f a^{k} / f a^{k}$ rats, Ad-LEPR-Btreated animals exhibited a $45 \%$ inhibition of food intake after CCK injection $(P<0.05)$ (Figure 3).

Experiment 3: Effect of leptin receptor deficiency and ARC-directed leptin receptor gene therapy on hindbrain c-Fos induction by CCK. To determine whether CCK-induced activation of hindbrain neurons is attenuated in leptin receptor-deficient rats and, if so, whether leptin receptor signaling localized to the vicinity of the ARC of these animals restores this response, we measured CCK-induced c-Fos expression in the hindbrains of both $\mathrm{Fa} / \mathrm{Fa}$ and $f a^{k} / f a^{k}$ animals that had previously received ARC-directed gene therapy. Compared with i.p. saline, CCK increased the mean number of nuclei that were positive for c-Fos-like immunoreactivity (cFLI) within both the AP and NTS of animals in each group. Although there was no difference in the estimated number of c-Fos-positive cells among saline-treated animals, the effect of CCK on increasing the cFLI-positive cell number was reduced in the hindbrains of $f a^{k} / f a^{k}$ rats compared with $\mathrm{Fa} / \mathrm{Fa}$ rats when both groups were treated with control adenovirus (Figure 4, A and B). Thus, $f a^{k} / f a^{k}$ rats treated with adenovirus expressing the reporter gene GFP (Ad-GFP) manifested a $32 \%$ reduction in the number of AP neurons that contained Fos compared with that of Fa/Fa controls (54.2 \pm 11.2 vs. $79.8 \pm 8.1$; $P<0.05)$, while a $35 \%$ reduction was detected in the NTS $(180 \pm 32$ vs. $278 \pm 10 ; P<0.05$ ) (Figure 4, A and B). Interestingly, the effect of CCK on increasing cFLI-positive neurons in $f a^{k} / \mathrm{fa}^{k}$ rats receiving ARC-directed Ad-LEPR-B-GFP was greater than that in $f a^{k} / f a^{k}$ animals receiving control adenovirus in both the AP $(86.0 \pm 3.8$ vs.
$54.2 \pm 11.2 ; P<0.05)$ and the NTS $(258 \pm 31$ vs. $180 \pm 32 ; P<0.05)$ and was not different from the response to CCK in $\mathrm{Fa} / \mathrm{Fa}$ rats receiving Ad-GFP (Figure 4, A and B). Representative images of cFLI in coronal sections of AP and NTS from animals in each of the 3 groups are presented in Figures 5 and 6.

To determine the anatomical distribution of adenoviral gene expression, we prepared brain sections for visualization of GFP by fluorescent microscopy after microinjection of Ad-GFP or Ad-LEPR-B-GFP in the animals described above (Figure 7). Seven days after microinjection of Ad-LEPR-B-GFP, GFP expression was concentrated within the ARC in 5 of 5 animals. GFP expression was additionally detected along the cannula tract, either just dorsal or ventral to the ARC, in 2 of these animals but was not detected in brain areas outside the hypothalamus (including the thalamus, cortex or hindbrain), nor was it present in cells lining the third ventricle. Of the 10 rats microinjected with Ad-GFP, 8 animals were positive for GFP expression in the ARC, while GFP expression was not detected in any brain area in 2 animals, despite visualization of the cannula tract descending into the ARC. In the remaining 8 animals, GFP was localized predominantly in the ARC. In 3 of these animals, GFP expression was additionally detected along the cannula tract, either just dorsal or ventral to the ARC, including a small number of GFP-positive cells detected in the ventromedial nucleus (VMN). Again, GFP was not detected in areas outside the hypothalamus.

\section{Discussion}

Abundant evidence supports the hypothesis that humoral negative feedback in proportion to body fat stores is communicated to brain areas controlling food intake and that this input is crucial for normal energy homeostasis $(7,8)$. Despite impressive progress in the identification of specific neurons and molecules that participate

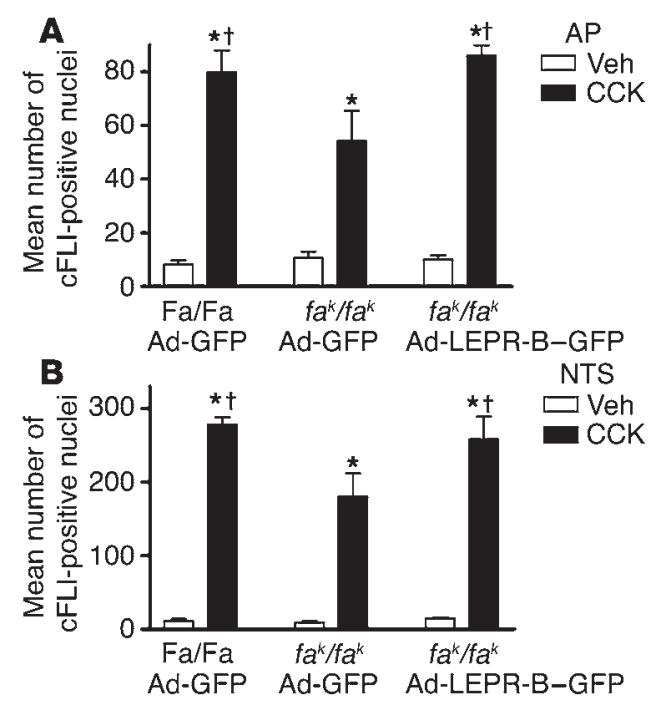

Figure 4

Effect of ARC-directed leptin receptor gene therapy on hindbrain c-Fos induction by CCK. Mean number of CFLI-positive nuclei per section in the AP (A) or NTS (B) as determined by immunohistochemistry after i.p. injection of either saline vehicle (white bars) or a 1.0- $\mu$ g dose of CCK (black bars) 7 days after adenoviral microinjection of either Ad-GFP (in $\mathrm{Fa} / \mathrm{Fa}$ or $\mathrm{fa}^{k} / \mathrm{fa}^{k}$ rats) or Ad-LEPR-B-GFP (in $f a^{k} / \mathrm{fa}^{k}$ rats). ${ }^{*} P<0.05$, vehicle vs. CCK; ${ }^{\dagger} P<0.05$, CCK treatment in Fa/Fa Ad-GFP and $f a^{k} / \mathrm{fa}^{k}$ Ad-LEPR-B-GFP vs. $f a^{k} / f a^{k}$ Ad-GFP-treated rats. 


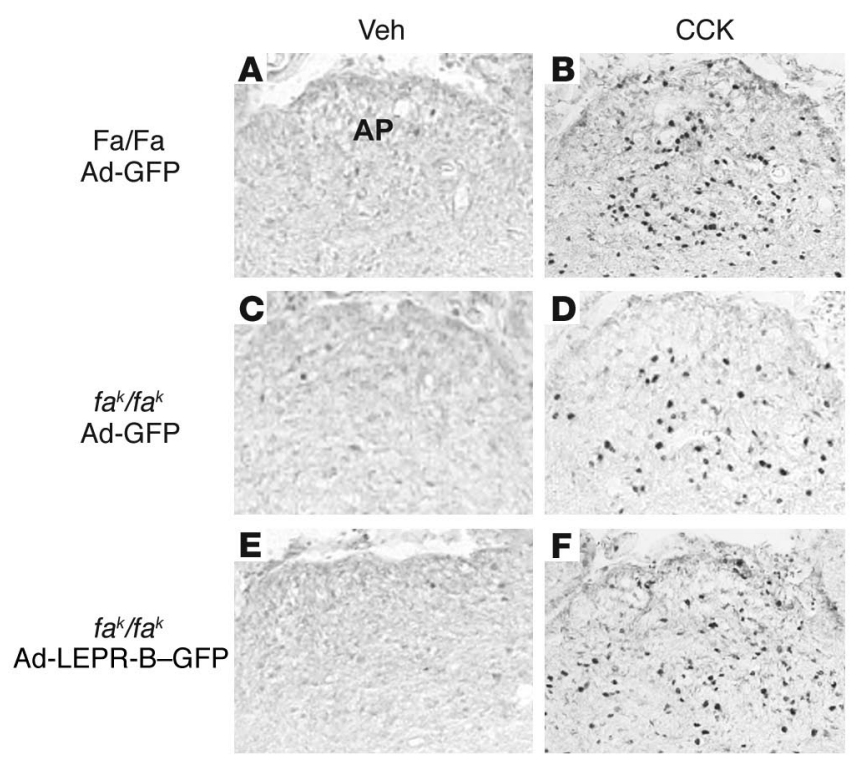

\section{Figure 5}

Induction of c-Fos at the level of the AP. Representative images of cFLI in coronal sections of rat hindbrain at the level of the AP in animals from each of 3 groups (Fa/Fa Ad-GFP; $f a^{k} / f a^{k}$ Ad-GFP; and $f a^{k} / f a^{k}$ Ad-LEPR-B-GFP) treated with either i.p. saline vehicle (A, C, and E) or a 1.0- $\mu \mathrm{g}$ dose of CCK (B, D, and $\mathbf{F})$.

in this process, the mechanism whereby long-term changes of body adiposity are coupled to adaptive short-term adjustments of energy intake remains poorly understood. To investigate the mechanisms linking leptin action in the CNS to adaptive changes of food intake, we compared genetically obese $f a^{k} / f a^{k}$ rats, which otherwise lack all leptin receptor protein, with their lean littermates. Our finding that hyperphagia of $f a^{k} / f a^{k}$ rats is due entirely to the consumption of larger meals, rather than to an increased number of meals, is consistent with results in other models of deficient leptin or leptin receptor signaling (33-35) and suggests that leptin signaling is a critical determinant of the ability of satiety signals to induce meal termination. Strengthening this conclusion is our finding that satiety and hindbrain responses to CCK are impaired in these leptin receptor-deficient animals.

Several potential mechanisms can be invoked to explain how the response to satiety signals is impaired by the absence of leptin signaling, thereby favoring the consumption of larger meals. Given the large body of literature implicating ARC neurons as targets for the action of leptin in energy homeostasis $(7,8)$, we and others have hypothesized that leptin signaling in this brain area is a critical determinant of sensitivity to satiety signals (36). Implicit in this hypothesis is a functional link between neurons in the ARC and those in caudal hindbrain areas such as the NTS, where input from satiety signals is processed (Figure 8). An alternative model proposes that the potentiating effect of leptin on CCK-induced satiety involves local actions of leptin on CCK-sensitive neurons within the hindbrain, as leptin receptors are expressed in the NTS $(28,29)$ and because leptin reduces food intake when infused directly into this brain area $(28,37,38)$. Although the question of whether local actions of leptin in the hindbrain can regulate the response to satiety signals remains untested and is an area of interest for future studies, our findings constitute direct evidence that leptin action in the forebrain modulates both meal size and the hindbrain response to CCK. This conclusion stems from our finding that increased meal size and impaired behavioral and hindbrain responses to CCK were partially or completely "rescued" by direction of expression of the long form of the leptin receptor to the ARC of $f a^{k} / f a^{k}$ rats. Although leptin receptor expression was also detected along the cannula tract just dorsal or ventral to the ARC in a minority of animals, the ARC was the predominant site of adenoviral gene expression detected after microinjection directed to the ARC. As expression was not detected in areas outside the hypothalamus, consistent with our previous findings (32), we conclude that responses induced by ARC-directed leptin receptor gene therapy are attributable to actions on ARC neurons and immediately adjacent portions of the mediobasal hypothalamus.

The potential of this model (Figure 8) in clarifying how afferent input from body energy stores influences food intake on a mealto-meal basis is its greatest strength. For example, it predicts that animals lacking this leptin signal should fail to terminate meals in response to normal satiety cues and hence overeat and develop massive obesity. Our finding that leptin receptor-deficient rodents consume excessively large meals and are less sensitive to CCK-induced satiety than lean littermates is in agreement with this prediction. Although this observation is compatible with previous evidence $(21-24,34,39,40)$ that leptin signaling is required for intact behavioral responses to meal-related satiety cues, it contrasts with the intact response to CCK reported in Koletsky rats by Wildman and colleagues (41). This discrepancy probably reflects differences in testing paradigms, as the satiating action of CCK in the previous report was tested by measuring intake of a sucrose solution instead of laboratory chow. As a result, rats of both genotypes consumed relatively large amounts of test solution and the hyperphagia generally characteristic of obese animals relative to

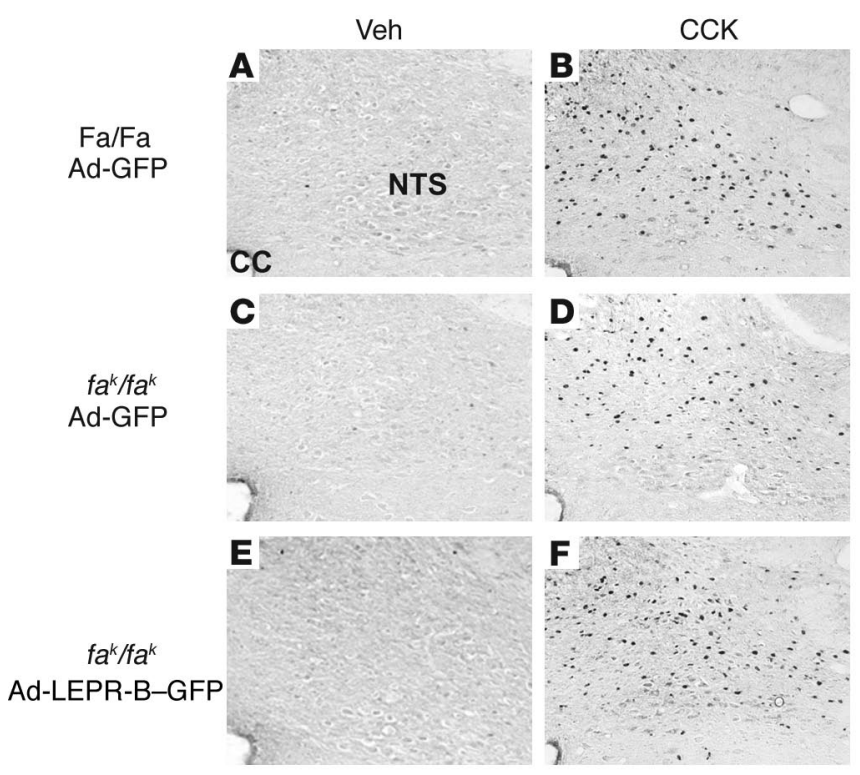

Figure 6

Induction of c-Fos at the level of the NTS. Representative images of cFLI in coronal sections of rat hindbrain at the level of the NTS in animals from each of 3 groups (Fa/Fa Ad-GFP; $f a^{k} / f a^{k}$ Ad-GFP; and $f a^{k} / f a^{k}$ Ad-LEPR-B-GFP) treated with either i.p. saline vehicle (A, C, and E) or a1.0- $\mu$ g dose of CCK (B, D, and F). CC, central canal. 
A
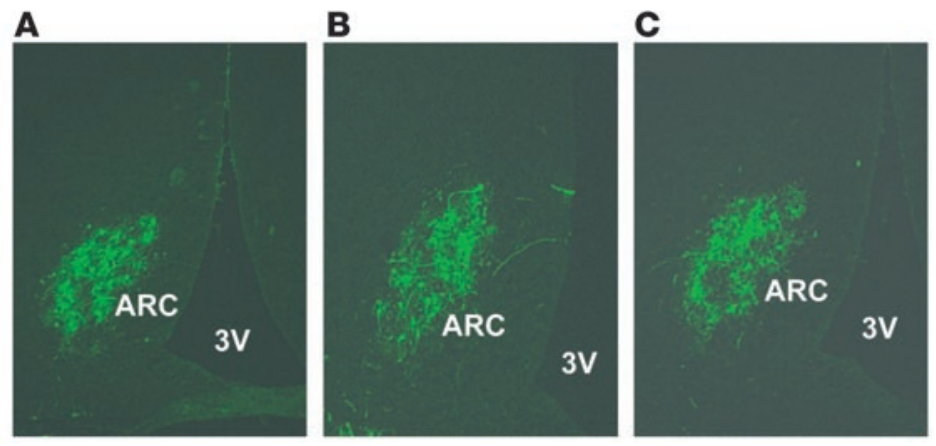

Figure 7

Detection of adenovirally expressed genes. Expression of GFP after microinjection of adenovirus expressing either GFP alone (Ad-GFP) in Fa/Fa (A) or $f a^{k} / f a^{k}(B)$ rats or both leptin receptor and GFP (Ad-LEPR-B-GFP) in $f a^{k} / f a^{k}$ rats $(\mathbf{C})$, visualized using fluorescence microscopy. Magnification, $\times 10.3 \mathrm{~V}$, third ventricle.

lean controls was not evident. Perhaps more importantly, Wildman et al. (41) used a relatively high dose of CCK, and our data suggest that differences between genotypes in the satiety response are minimized at high CCK doses. Combined with our findings that CCK-induced c-Fos expression is reduced by approximately $35 \%$ in the NTS and AP of rats lacking leptin receptors, we conclude that leptin signaling is required for intact responses of key hindbrain neurons to this satiety peptide. As the attenuated hindbrain response to i.p. CCK was restored by ARC-directed leptin receptor expression, our data further suggest that leptin signaling in this hypothalamic area is a key determinant of the hindbrain response to input from meal-related satiety signals.

Although the mechanism whereby the hindbrain response to CCK is regulated by leptin-sensing hypothalamic neurons remains to be firmly established, it probably involves connections between ARC neurons and adjacent hypothalamic subnuclei (e.g., the paraventricular nucleus [PVN] and lateral hypothalamic area [LHA]) $(42,43)$ that, in turn, project directly to the NTS, although fibers projecting directly from ARC neurons to the hindbrain have also been described. In general, neurons situated in the LHA are orexigenic in nature $(44,45)$ (and hence are predicted to blunt the hindbrain response to satiety signals and to be inhibited by leptin-generated signals in the ARC), while the opposite is true for PVN neurons implicated in this neurocircuit $(46,47)$. Among PVN neurons with the potential to convey anorexigenic, leptin-generated signals to the NTS are those that contain oxytocin. This assertion is based on the close anatomical proximity between oxytocin-containing fibers projecting from the PVN and CCK-activated neurons in the NTS (48) and on evidence that these oxytocin-producing PVN neurons are activated by leptin (27). Further, the ability of leptin to potentiate CCK-induced activation of NTS neurons appears to require oxytocin signaling, as it is attenuated by pretreatment with an oxytocin receptor antagonist (27). These findings support a model in which descending oxytocinergic projections are among several hypothalamic cell groups that convey leptin-generated neuronal responses to hindbrain areas that respond to satiety signals.

Within the ARC are 2 well described neuronal subpopulations that express leptin receptors $(49,50)$, potently regulate food intake, and have well defined projections to both the PVN and LHA $(43,51)$. One subpopulation is comprised of orexigenic neurons that con- tain neuropeptide Y (NPY) and agouti-related peptide (AgRP) (52-54) and are inhibited by leptin $(2,5)$, while the other comprises anorexigenic neurons that express proopiomelanocortin (POMC), the melanocortin precursor peptide, and are stimulated by leptin $(3,4,10)$. Together with inhibition of NPY/AgRP neurons, activation of POMC neurons is strongly implicated in the mechanism whereby leptin reduces food intake, and growing evidence supports the idea that both neuronal subsets have the potential to regulate the response of NTS neurons to input from satiety signals. For example, icv infusion of NPY increases meal size (55) and attenuates both satiety and hindbrain responses to CCK (24) while also reducing the sensitivity of hindbrain neurons to other satiety-inducing stimuli such as gastric distension (56). Conversely, injection of the melanocortin $\alpha-\mathrm{MSH}$ directly into the PVN reduces meal size (57), and signaling via neuronal melanocortin-4 receptors, which mediate the anorectic actions of $\alpha-\mathrm{MSH}$, is required for CCK-induced suppression of feeding (58). Increased meal size in rats lacking leptin receptors may therefore arise, at least in part, from increased signaling by NPY and AgRP, combined with reduced signaling by melanocortins $(3,6,8,50)$. Consistent with this hypothesis is our recent report that restoration of leptin signaling to the ARC of $f a^{k} / f a^{k}$ rats lowers Npy mRNA while increasing Pomc gene expression in this brain area (32).

Because CCK-induced satiety was not fully normalized by restoration of hypothalamic leptin signaling in $f a^{k} / f a^{k}$ rats, additional brain areas and signaling systems are likely to mediate interactions between leptin and CCK. As noted above, actions of leptin on neurons in the NTS or other leptin-sensitive brain areas (e.g., hypothalamic ventromedial or dorsomedial nuclei) may also participate in this process, in keeping with a "distributed" model

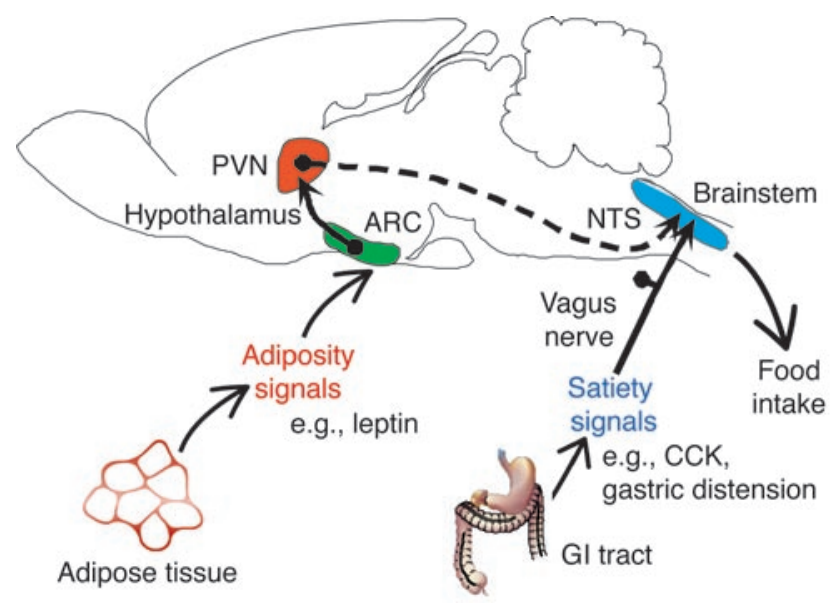

Figure 8

Model for regulation of the hindbrain response to satiety signals by hormonal input from the ARC. Adiposity signals such as insulin and leptin circulate in proportion to body fat mass and act on hypothalamic ARC neurons that project to hypothalamic areas such as the LHA (not shown) and PVN. In turn, these "second order" neurons project to hindbrain autonomic centers such as the NTS that process afferent input from satiety signals such as CCK. Input from descending, leptin-sensitive hypothalamic projections is integrated in the NTS with vagally mediated input from CCK, such that the timing of meal termination is regulated by changes in body fat content. Modified with permission from Nature (7). GI, gastrointestinal. 
of neural networks that process and integrate adiposity- and satiety-related inputs (14). A related and paradoxical finding is that among $f a^{k} / f a^{k}$ rats treated with a control adenovirus, CCKinduced activation of NTS neurons was reduced by only $35 \%$, whereas the satiety response to the same dose of CCK was markedly attenuated. Thus, CCK activates neurons in the hindbrains of $f a^{k} / f a^{k}$ rats at a dose that does not have a reliable food intake effect. Many NTS and AP neurons can therefore respond to vagally transmitted signals via a mechanism that is independent of leptin signaling and is potentially unrelated to the perception of satiety. Accordingly, it seems likely that CCK activates neurons in the hindbrain of $f a^{k} / f a^{k}$ rats that participate in physiological functions unrelated to satiety, or that under certain conditions, activation of hindbrain neurons (as judged by cFLI) can occur in the absence of a measurable physiological response. These observations also raise the possibility that leptin signaling in the forebrain regulates the response of an as-yet-unidentified subset of neurons involved in satiety that are located downstream of CCK-activated neurons in the NTS/AP. The phenotypic characterization of hindbrain neuronal subsets that respond to satiety signals via a mechanism that is sensitive to input from leptinactivated pathways (and those that do not) is an interesting challenge for future studies.

In conclusion, we have reported here that intact leptin signaling is required for proper processing of satiety-related cues and for normal meal termination and that restoration of leptin receptor signaling in the area of the ARC of $f a^{k} / f a^{k}$ rats reduces meal size and enhances CCK-induced satiety and activation of hindbrain neurons. Collectively, these findings support the hypothesis that neurons in forebrain areas regulate food intake in response to afferent hormonal input, at least in part, by controlling the response of hindbrain areas that process input from meal-related satiety signals. Signaling via this mechanism provides a compelling explanation for how changes of body adiposity are linked to adaptive adjustments of energy intake during individual meals.

\section{Methods}

\section{Animals}

Male obese $\left(f a^{k} / f a^{k}\right)$ Koletsky rats and lean $(\mathrm{Fa} / \mathrm{Fa})$ littermates were generated from serial backcrosses (N10 equivalent) of the $\mathrm{fa}^{k}$ mutation (also known as Koletsky, $f a f, f$, or $c p$ ) to the inbred rat strain LA/N. All animals were housed individually in microisolator polycarbonate cages under specific-pathogen free conditions, maintained in a temperature-controlled room with a 12-hour/12-hour light/dark cycle and were provided with ad libitum access to standard laboratory chow (PMI Nutrition International Inc.) and water. All procedures were conducted in 16- to 20-week-old animals and were approved by the Animal Care and Use Committee at the University of Washington and performed in accordance with NIH Guidelines for the Care and Use of Animals.

\section{Meal pattern analysis}

To determine the size and number of meals consumed by $f a^{k} / f a^{k}$ rats relative to their $\mathrm{Fa} / \mathrm{Fa}$ littermates, we housed animals for 24 hours in calorimeter chambers equipped with the Feed-Scale System (Columbus Instruments) designed to continuously measure the rate at which food is consumed. Meal pattern analysis was performed using the following criteria. The size of an individual meal was defined as the consumption of at least $0.2 \mathrm{~g}$ of chow that was separated from the end of the previous meal by at least 15 minutes. Based on these criteria, we subsequently analyzed 24-hour food intake data using a computer program (Visual Basic) and found that defining meal criteria in this way accounted for over $99 \%$ of total daily food intake. Meal duration was defined as the time from the beginning to the end of a single meal, and the intermeal interval, as the time from the end of one meal to the beginning of the next. Average meal size was calculated by dividing the number of feeding bouts (individual meals) by the total amount of food consumed (in grams) over the test period ( 24 hours). Varying these criteria by 1 order of magnitude did not alter the pattern of differences observed between meal-related parameters across study groups.

\section{Determination of CCK-induced satiety}

Prior to the study, male $f a^{k} / f a^{k}$ and $\mathrm{Fa} / \mathrm{Fa}$ rats were habituated to regular handling and i.p. saline injections for 1 week. Immediately prior to the dark cycle onset and after a 4-hour fast, either sulfated CCK octapeptide (Peninsula Laboratories Inc.) at a dose of $1.0 \mu \mathrm{g}$ or saline vehicle was injected i.p. in a final volume of $2.0 \mathrm{ml}$. Food consumption was measured for 30 minutes after injections (i.e., during the first 30 minutes of the dark cycle onset); the value reported for each animal represents the mean of 3 replicate studies. The selection of the CCK dose was based on a dose-response study in which the 30-minute feeding response was measured after i.p. administration of CCK at doses between 0.75 and $5 \mu \mathrm{g}$ or of saline vehicle at the onset of the dark cycle. This study revealed an unambiguous difference in the response of $f a^{k} / f a^{k}$ and $\mathrm{Fa} / \mathrm{Fa}$ rats to the $1-\mu \mathrm{g}$ dose of CCK but not to a higher dose of CCK $(5 \mu \mathrm{g})$.

\section{Generation and microinjection of recombinant adenovirus}

Ad-lac $Z$ and human leprb adenovirus were generated and purified as described previously $(59,60)$. Animals were placed in a stereotaxic frame (Cartesian Research Inc.) after being sedated with isofluorane anaesthesia, and the ARC was targeted bilaterally via 2 unilateral injections using a microinfusion pump (UMPII; World Precision Instruments Ltd.) and a 28-guage syringe system (Hamilton Syringes) directed to the stereotaxic coordinates $2.8 \mathrm{~mm}$ posterior to bregma, $\pm 0.25 \mathrm{~mm}$ lateral to the midline, and $10.5 \mathrm{~mm}$ below the surface of the skull. Adenovirus expressing either lepr $^{b}\left(2.4 \times 10^{12} \mathrm{PFU} / \mathrm{ml}\right)$ or lacZ $\left(1.7 \times 10^{12} \mathrm{PFU} / \mathrm{ml}\right)$ was used for behavioral studies. We have previously shown that this microinjection procedure results in the expression of functional $\beta$-galactosidase, the product encoded by lacZ, or long-form leptin receptor proteins in a distribution that is localized to the ARC, with additional expression along the cannula tract occurring in less than $25 \%$ of injections (32). Injection of Ad-lacZ into the ARC according to this protocol was without adverse behavioral effects and did not alter daily food intake or body weight compared with that of saline-injected controls (32). For the histochemical study (experiment 3), an adenoviral construct containing lepr $r^{b}$ that also expresses the reporter gene GFP (Ad-LEPR-B-GFP; $3.4 \times 10^{12} \mathrm{PFU} / \mathrm{ml}$ ) was used. The adenoviral control for this study expresses GFP alone (Ad-GFP; $2.7 \times 10^{12} \mathrm{PFU} / \mathrm{ml}$ ). Adenovirus was administered to rats at a rate of 100 nanoliters per minute for 5 minutes ( $500 \mathrm{nl}$ per injection site), and the entire injector system and cannulae were removed 5 minutes after the injections were completed.

\section{Study protocols}

Experiment 1. Effect of leptin receptor deficiency and ARC-directed leptin receptor gene therapy on meal patterns. To determine if the hyperphagia of $f a^{k} / f a^{k}$ rats arises from increased size or number of meals (or both) relative to that of their lean littermates, we measured food intake continuously over a 24hour period in rats of both genotypes ( $n=4 /$ group) and performed meal pattern analysis. The effect of leptin receptor gene therapy directed to the ARC on feeding behavior was determined by performing meal pattern analysis 6 days after microinjection of either Ad-LEPR-B or Ad-lacZ into the ARCs of $f a^{k} / f a^{k}$ rats $(n=5 /$ group). 
Experiment 2. Effect of leptin receptor deficiency and ARC-directed leptin receptor gene therapy on CCK-induced satiety. To determine if leptin receptor deficiency is associated with an impaired satiety response to CCK and if leptin receptor gene therapy reverses this effect, we studied $f a^{k} / f a^{k}$ rats 6 days after microinjection into the ARCs of either Ad-LEPR-B $(n=6)$ or Ad-lacZ $(n=6)$. After a 4-hour fast, the effect of sulfated CCK octapeptide on 30-minute food consumption relative to intake after i.p. saline injection was determined at a time of day corresponding to the onset of the dark cycle, as described above. The feeding response of both groups of $f a^{k} / f a^{k}$ rats was compared with that of $\mathrm{Fa} / \mathrm{Fa}$ controls that received Ad-lac $\mathrm{Z}$ microinjection into the ARC and were subsequently treated with the same CCK dose. The value reported for each animal represents the mean of 3 replicates.

Experiment 3. Effect of leptin receptor deficiency and ARC-directed leptin receptor gene therapy on hindbrain c-Fos induction by CCK. To determine whether the ability of CCK to activate hindbrain neurons is attenuated by leptin receptor deficiency and whether this effect is reversed by leptin receptor gene therapy directed to the ARC, we used a quantitative cell counting method to detect cFLI-positive cells in the NTS and AP of 3 groups of rats, each of which received bilateral microinjection of adenovirus targeting the $\operatorname{ARC}\left(n=5 /\right.$ group): (a) Fa/Fa rats receiving Ad-GFP; (b) $f a^{k} / f a^{k}$ rats receiving Ad-GFP; and (c) $f a^{k} / f a^{k}$ rats microinjected with Ad-LEPR-B-GFP. Seven days after microinjection, animals were made to fast overnight and each subsequently received an i.p. injection of either saline or CCK $(1.0 \mu \mathrm{g})$. These injections were given 4 hours prior to the dark cycle onset and food was withheld after injections to avoid potential confounding effects of feeding on hindbrain c-Fos expression. Ninety minutes after injection of saline or CCK, rats were anaesthetized with ketamine/xylazine and were perfused with phosphate-buffered saline (PBS) followed by $4 \%$ paraformaldehyde (PFA) in $0.1 \mathrm{M}$ PBS. Brains were removed, post-fixed in $4 \%$ PFA overnight, embedded in $25 \%$ sucrose solution for 48 hours at $4{ }^{\circ} \mathrm{C}$, "snap-frozen" in isopentane, and cooled with liquid nitrogen. Both the hindbrain and hypothalamus were sectioned at $14 \mu \mathrm{m}$ in a freezing cryotome, collected on slides, and stored at $-80^{\circ} \mathrm{C}$ before assay for $\mathrm{CFLI}$ as described below or for visualization of GFP by fluorescence microscopy.

\section{c-Fos immunohistochemistry}

Coronal cryostat sections $(14 \mu \mathrm{m})$ of rat brain were warmed to room temperature and were equilibrated in $0.1 \mathrm{M}$ PBS. For each animal, 3 slides (6 sections) were selected from a defined region of the caudal hindbrain that contains both the AP and NTS (bregma $-5.231 \mathrm{~mm}$ ). Slides were subsequently processed for cFLI as described in detail elsewhere (61) and all slides were stained in a single assay. Histochemical images were captured using a Nikon Eclipse E600 upright microscope equipped with a Diagnostic Instruments Spot RT Color digital camera. The number of cFLI-positive cell nuclei was quantified in anatomically matched brain sections by an investigator "blinded" to study conditions. Quantification was determined using a computer-based image analysis system (Scion Corp.) by setting both a threshold value and a minimum particle size. Values generated using this approach were validated by comparison with cell counts obtained visually (data not shown). The mean number of cFLI-positive cells reported for the bilateral NTS and the AP of each animal was determined from 6 anatomically matched sections per animal.

\section{Statistical analysis}

All results are expressed as mean \pm SEM. Comparisons between multiple groups were made using a 1-way analysis of variance with a least significant difference post-hoc test for comparisons between groups. For 2-group comparisons, a 2-sample, unpaired Student's $t$ test was used, while a paired Student's $t$ test was used for within-group comparisons. Statistical analyses were performed using Statistical Package for the Social Sciences (SPSS, Version 10.1; SPSS Inc.). Probability values of less than 0.05 were considered significant.

\section{Acknowledgments}

The authors recognize the excellent technical assistance provided by Hong Nguyen and Loan Nguyen and thank Kayoko Ogimoto and Joost Overduin for support in the analysis of meal patterns. This work was supported by NIH grants DK52989, DK12829, DK68340, and NS32273 (to M.W. Schwartz) and by the Diabetes Endocrinology Research Center and Clinical Nutrition Research Unit of the University of Washington. G.J. Morton is supported by a mentor-based fellowship from the American Diabetes Association.

Received for publication May 5, 2004, and accepted in revised form December 22, 2004.

Address correspondence to: Michael Schwartz, Department of Medicine, Harborview Medical Center, University of Washington, 325 Ninth Avenue, Box 359675, Seattle, Washington 98104, USA. Phone: (206) 341-5288; Fax: (206) 341-5293; E-mail: mschwart@u.washington.edu.
1. Zhang, Y., et al. 1994. Positional cloning of the mouse obese gene and its human homologue. Nature. 372:425-432.

2. Schwartz, M.W., et al. 1996. Specificity of leptin action on elevated blood glucose levels and hypothalamic neuropeptide $\mathrm{Y}$ gene expression in $\mathrm{ob} / \mathrm{ob}$ mice. Diabetes. 45:531-535.

3. Schwartz, M.W., et al. 1997. Leptin increases hypothalamic pro-opiomelanocortin mRNA expression in the rostral arcuate nucleus. Diabetes. 46:2119-2123.

4. Thornton, J.E., Cheung, C.C., Clifton, D.K., and Steiner, R.A. 1997. Regulation of hypothalamic proopiomelanocortin mRNA by leptin in ob/ob mice. Endocrinology. 138:5063-5066.

5. Mercer, J.G., Moar, K.M., Rayner, D.V., Trayhurn, P., and Hoggard, N. 1997. Regulation of leptin receptor and NPY gene expression in hypothalamus of leptin-treated obese (ob/ob) and cold-exposed lean mice. FEBS Lett. 402:185-188.

6. Mizuno, T.M., et al. 1998. Hypothalamic pro-opiomelanocortin mRNA is reduced by fasting in $\mathrm{ob} / \mathrm{ob}$ and $\mathrm{db} / \mathrm{db}$ mice, but is stimulated by leptin.
Diabetes. 47:294-297.

7. Schwartz, M.W., Woods, S.C., Porte, D., Jr., Seeley, R.J., and Baskin, D.G. 2000. Central nervous system control of food intake. Nature. 404:661-671.

8. Saper, C.B., Chou, T.C., and Elmquist, J.K. 2002. The need to feed: homeostatic and hedonic control of eating. Neuron. 36:199-211.

9. Zigman, J.M., and Elmquist, J.K. 2003. Minireview: From anorexia to obesity--the yin and yang of body weight control. Endocrinology. 144:3749-3756.

10. Cowley, M.A., et al. 2001. Leptin activates anorexigenic POMC neurons through a neural network in the arcuate nucleus. Nature. 411:480-484.

11. Spanswick, D., Smith, M.A., Groppi, V.E., Logan, S.D., and Ashford, M.L. 1997. Leptin inhibits hypothalamic neurons by activation of ATP-sensitive potassium channels. Nature. 390:521-525.

12. Strubbe, J.H., and Woods, S.C. 2004. The timing of meals. Psychol. Rev. 111:128-141.

13. Gibbs, J., Young, R.C., and Smith, G.P. 1973. Cholecystokinin decreases food intake in rats. J. Comp. Physiol. Psychol. 84:488-495.

14. Grill, H.J., and Kaplan, J.M. 2002. The neuroana- tomical axis for control of energy balance. Front. Neuroendocrinol. 23:2-40.

15. Kahler, A., et al. 1998. Chronic administration of $\mathrm{OB}$ protein decreases food intake by selectively reducing meal size in male rats. Am. J. Physiol. 275:R180-R185.

16. Eckel, L.A., et al. 1998. Chronic administration of $O B$ protein decreases food intake by selectively reducing meal size in female rats. Am. J. Physiol. 275:R186-R193.

17. Flynn, M.C., Scott, T.R., Pritchard, T.C., and PlataSalaman, C.R. 1998. Mode of action of OB protein (leptin) on feeding. Am. J. Physiol. 275:R174-R179.

18. Hulsey, M.G., Lu, H., Wang, T., Martin, R.J., and Baile, C.A. 1998. Intracerebroventricular (i.c.v.) administration of mouse leptin in rats: behavioral specificity and effects on meal patterns. Physiol. Behav. 65:445-455.

19. Barrachina, M.D., Martinez, V., Wang, L., Wei, J.Y., and Tache, Y. 1997. Synergistic interaction between leptin and cholecystokinin to reduce short-term food intake in lean mice. Proc. Natl. Acad. Sci. U. S. A. 94:10455-10460. 
20. Emond, M., Schwartz, G.J., Ladenheim, E.E., and Moran, T.H. 1999. Central leptin modulates behavioral and neural responsivity to CCK. Am. J. Physiol. 276:R1545-R1549.

21. Wang, L., Martinez, V., Barrachina, M.D., and Tache, Y. 1998. Fos expression in the brain induced by peripheral injection of CCK or leptin plus CCK in fasted lean mice. Brain Res. 791:157-166.

22. McLaughlin, C.L., and Baile, C.A. 1980. Decreased sensitivity of Zucker obese rats to the putative satiety agent cholecystokinin. Physiol. Behav. 25:543-548.

23. McLaughlin, C.L., Peikin, S.R., and Baile, C.A. 1983. Food intake response to modulation of secretion of cholecystokinin in Zucker rats. Am. J. Physiol. 244:R676-R685.

24. McMinn, J.E., Sindelar, D.K., Havel, P.J., and Schwartz, M.W. 2000. Leptin deficiency induced by fasting impairs the satiety response to cholecystokinin. Endocrinology. 141:4442-4448.

25. Woods, S.C. 2004. Gastrointestinal Satiety Signals I. An overview of gastrointestinal signals that influence food intake. Am. J. Physiol. Gastrointest. Liver Physiol. 286:G7-G13.

26. Woods, S.C., Seeley, R.J., Porte, D., Jr., and Schwartz, M.W. 1998. Signals that regulate food intake and energy homeostasis. Science. 280:1378-1383.

27. Blevins, J.E., Schwartz, M.W., and Baskin, D.G. 2004. Evidence that paraventricular nucleus oxytocin neurons link hypothalamic leptin action to caudal brainstem nuclei controlling meal size. Am. J. Physiol. Regul. Integr. Comp. Physiol. 287:R87-R96.

28. Grill, H.J., et al. 2002. Evidence that the caudal brainstem is a target for the inhibitory effect of leptin on food intake. Endocrinology. 143:239-246.

29. Mercer, J.G., et al. 1996. Localization of leptin receptor mRNA and the long form splice variant (Ob-Rb) in mouse hypothalamus and adjacent brain regions by in situ hybridization. FEBS Lett. 387:113-116.

30. Wu-Peng, X.S., et al. 1997. Phenotype of the obese Koletsky (f) rat due to Tyr763Stop mutation in the extracellular domain of the leptin receptor (Lepr): evidence for deficient plasma-to-CSF transport of leptin in both the Zucker and Koletsky obese rat. Diabetes. 46:513-518.

31. Takaya, K., et al. 1996. Nonsense mutation of leptin receptor in the obese spontaneously hypertensive Koletsky rat. Nat. Genet. 14:130-131.

32. Morton, G.J., et al. 2003. Arcuate nucleus-specific leptin receptor gene therapy attenuates the obesity phenotype of Koletsky $(\mathrm{fa}(\mathrm{k}) / \mathrm{fa}(\mathrm{k}))$ rats. Endocrinology. 144:2016-2024.

33. Azzara, A.V. 2004. Genetic and biobehavioral analysis of the controls of meal size in mice. Appetite.
42:107-109.

34. Becker, E.E., and Grinker, J.A. 1977. Meal patterns in the genetically obese Zucker rat. Physiol. Behav. 18: $685-692$.

35. Strohmayer, A.J., and Smith, G.P. 1987. The meal pattern of genetically obese $(\mathrm{ob} / \mathrm{ob})$ mice. Appetite. 8:111-123.

36. Schwartz, G.J., and Azzara, A.V. 2004. Sensory neurobiological analysis of neuropeptide modulation of meal size. Physiol. Behav. 82:81-87.

37. Hosoi, T., Kawagishi, T., Okuma, Y., Tanaka, J., and Nomura, Y. 2002. Brain stem is a direct target for leptin's action in the central nervous system. Endocrinology. 143:3498-3504.

38. Mercer, J.G., Moar, K.M., and Hoggard, N. 1998 Localization of leptin receptor (Ob-R) messenger ribonucleic acid in the rodent hindbrain. Endocrinology. 139:29-34.

39. McLaughlin, C.L., and Baile, C.A. 1981. Ontogeny of feeding behavior in the Zucker obese rat. Physiol. Behav. 26:607-612.

40. Ho, A., and Chin, A. 1988. Circadian feeding and drinking patterns of genetically obese mice fed solid chow diet. Physiol. Behav. 43:651-656.

41. Wildman, H.F., Chua, S., Jr., Leibel, R.L., and Smith, G.P. 2000. Effects of leptin and cholecystokinin in rats with a null mutation of the leptin receptor Lepr(fak). Am. J. Physiol. Regul. Integr. Comp. Physiol. 278:R1518-R1523.

42. Elmquist, J.K. 2001. Hypothalamic pathways underlying the endocrine, autonomic, and behavioral effects of leptin. Physiol. Behav. 74:703-708.

43. Elias, C.F., et al. 1998. Chemically defined projections linking the mediobasal hypothalamus and the lateral hypothalamic area. J. Comp. Neurol. 402:442-459.

44. Qu, D., et al. 1996. A role for melanin-concentrating hormone in the central regulation of feeding behaviour. Nature. 380:243-246.

45. Sakurai, T., et al. 1998. Orexins and orexin receptors: a family of hypothalamic neuropeptides and $\mathrm{G}$ protein-coupled receptors that regulate feeding behavior. Cell. 92:573-585.

46. Rothwell, N.J. 1990. Central effects of CRF on metabolism and energy balance. Neurosci. Biobehav. Rev. 14:263-271.

47. Arletti, R., Benelli, A., and Bertolini, A. 1990. Oxytocin inhibits food and fluid intake in rats. Physiol. Behav. 48:825-830.

48. Blevins, J.E., Eakin, T.J., Murphy, J.A., Schwartz, M.W., and Baskin, D.G. 2003. Oxytocin innervation of caudal brainstem nuclei activated by cholecystokinin. Brain Res. 993:30-41.

49. Cheung, C.C., Clifton, D.K., and Steiner, R.A.
1997. Proopiomelanocortin neurons are direct targets for leptin in the hypothalamus. Endocrinology. 138:4489-4492.

50. Baskin, D.G., Breininger, J.F., and Schwartz, M.W. 1999. Leptin receptor mRNA identifies a subpopulation of neuropeptide $Y$ neurons activated by fasting in rat hypothalamus. Diabetes. 48:828-833.

51. Elias, C.F., et al. 1999. Leptin differentially regulates NPY and POMC neurons projecting to the lateral hypothalamic area. Neuron. 23:775-786.

52. Rossi, M., et al. 1998. A C-terminal fragment of Agouti-related protein increases feeding and antagonizes the effect of alpha-melanocyte stimulating hormone in vivo. Endocrinology. 139:4428-4431.

53. Hagan, M.M., et al. 2000. Long-term orexigenic effects of AgRP-(83-132) involve mechanisms other than melanocortin receptor blockade. Am. J. Physiol. Regul. Integr. Comp. Physiol. 279:R47-R52.

54. Stanley, B.G., Kyrkouli, S.E., Lampert, S., and Leibowitz, S.F. 1986. Neuropeptide Y chronically injected into the hypothalamus: a powerful neurochemical inducer of hyperphagia and obesity. Peptides. 7:1189-1192.

55. Leibowitz, S.F., and Alexander, J.T. 1991. Analysis of neuropeptide Y-induced feeding: dissociation of Y1 and Y2 receptor effects on natural meal patterns. Peptides. 12:1251-1260.

56. Schwartz, G.J., and Moran, T.H. 2002. Leptin and neuropeptide $\mathrm{Y}$ have opposing modulatory effects on nucleus of the solitary tract neurophysiological responses to gastric loads: implications for the control of food intake. Endocrinology. 143:3779-3784.

57. Wirth, M.M., Olszewski, P.K., Yu, C., Levine, A.S., and Giraudo, S.Q. 2001. Paraventricular hypothalamic alpha-melanocyte-stimulating hormone and MTII reduce feeding without causing aversive effects. Peptides. 22:129-134.

58. Fan, W., et al. 2004. Cholecystokinin-mediated suppression of feeding involves the brainstem melanocortin system. Nat. Neurosci. 7:335-336

59. Dickson, L.M., et al. 2001. Differential activation of protein kinase $\mathrm{B}$ and $\mathrm{p} 70(\mathrm{~S} 6) \mathrm{K}$ by glucose and insulin-like growth factor 1 in pancreatic beta-cells (INS-1). J. Biol. Chem. 276:21110-21120.

60. Skelly, R.H., Wicksteed, B., Antinozzi, P.A., and Rhodes, C.J. 2001. Glycerol-stimulated proinsulin biosynthesis in isolated pancreatic rat islets via adenoviral-induced expression of glycerol kinase is mediated via mitochondrial metabolism. Diabetes. 50:1791-1798.

61. Thiele, T.E., et al. 1998. Central infusion of melanocortin agonist MTII in rats: assessment of c-Fos expression and taste aversion. Am. J. Physiol. 274:R248-R254. 